\title{
Subject Index Vol. 11, 1975
}

Amphibians 161 Anterograde degeneration 257 Apes 48

Apomorphine 365 Auditory system 322

Binocular receptive field organization Brain 48, 179

- organisation 73

- size 73

- stimulation 109

Cerebellum 109 Cerebral asymmetries 48

- evolution 214

Chick embryo 130

Columbia Una 91

Columnar hypothesis 16

Cortical lesions 257, 275

Curiosity 1

Cutaneous nerves 60

Development 73, 130 Dipodomys merriami 322 Domestic cat 16 Dopamine 365

Endocranial casts 214 Evolution 179 Excitatory processes 130 Eye movements 109

Falling 53 Felid brains 214 Foramen magnum 1 Fossils 214 Fossil species 1

Glycine 130 Goldfish 109 
Haloperidol 365

Hippocampus 355 Hominids 73

Inhibitory processes 130 Insectivores 179161 Interlaminar connections 257

Kangaroo rat 322

Laminar thermocoagulation 275 Learning 355 - set 1

Macaque monkey 16

Man 1, 73, 179

Maturation 53

Medial longitudinal fasciculus 109

Medulla 1

Memory 355

Modality 16

Neocortex 1 Neuroregeneration 60 Neurospecificity 60

Oculomotor complex 109 Optic tectum 161

Pattern discrimination 275 Pigeon 91

Prefrontal cortex 355 Primates 1, 73 Prosimians 179

Rat 53, 257, 275 Receptive field 16 Reptiles 365 Righting reflex 53

Simians 179 


\section{Subject Index}

Somatosensory cerebral cortex 16

Spinal cord 130

Squirrel monkey 355

Stereotyped behavior 365

Strychnine 130

Sunfish 109

Superior olivary complex 322

Tectotectal relationships 91 Topographical hypothesis 16 
Tortoise 365 Trapezoid body 322 Trifluperazine 365 Trochlear nerve 109

Ventral cochlear nucleus 322 Vestibular nuclei 109 Visual cortex 257, 275 system 91 